\title{
Is It Possible to Innovate Work Organization? Ideas and Inspirations from Coworking Experiences
}

\author{
DOMENICO BERDICCHIA \\ domenico.berdicchia@unife.it \\ University of Ferrara
}

FULVIO FORTEZZA

fulvio.fortezza@unife.it

University of Ferrara

\begin{abstract}
Coworking is a recent phenomenon, but it is spreading and, even more importantly, it is evolving rapidly. It originated as a relatively simple way for freelancers to share work spaces, services and equipment in order to reduce individual costs. In most advanced cases, coworking organizations are turning into organizational settings in which truly novel work arrangements provide interesting new ideas and inspirations about how to bring about not only innovation in job design and work organization, but also a new way to construct individual and organizational identity. In this paper we will briefly describe how most advanced coworking organizations function, and we will reflect on some key elements that characterize them. We will also discuss about whether, and at what conditions, traditional companies and organizations can learn from such experiences.
\end{abstract}

\section{Introduction}

In the last few decades work evolved rapidly. Even without resorting to the common rhetoric about the presumed transition between Fordist and post-Fordist eras, there is little doubt that many significant aspects of work are changing at a fast pace. Even Greg Oldham and Richard Hackman, decades after their widely known publications that deeply influenced the mainstream literature and job design practices, in 2010 admitted that the 'object' of their studies (work) had deeply changed over time, and the theoretical models that seemed to be adequate in the past (including their own famous model) need to be deeply revised (Oldham and Hackman, 2010).

Work is now less constrained by time and space than ever before. It is increasingly varied, often temporary even in structured contexts, it crosses boundaries between units and organisations, it is less related to hierarchies and formal roles, it is strongly dependent on the evolution of technology. Most of all, work is becoming hard to define in terms of standard tasks and activities. Obviously, this is more or less significant depending on specific contexts 
and sectors, but these are undoubtedly the general trends that can be observed almost everywhere, across industries and countries.

While work has changed significantly, many of the deeper questions and issues about it seem to remain the same. Alienation, dissatisfaction, motivation, turnover, absenteeism, performance are the general, classic themes that Oldham and Hackman (2010) mention. We could add a few more: well-being, creativity, smart technologies, identity. Thus, while the phenomenon of work evolves, old issues need to be revisited, while new ones also emerge.

One might argue that the relationship between major managerial/organisational trends and work may be less 'unidirectional' than one might expect. The traditional view is that work changes are dependent on the strategic/organisational/technological choices by companies, especially multinational ones. However, this is not always the case. Coworking, the phenomenon we are focusing on in this article, seems to be an example of a transformation whose origins may originate, among other things, from an increasingly widespread tension and need to find new meaning and identity in work, a need that is not satisfied by traditional jobs in traditional organisations. The general thesis of this paper is that advanced coworking experiences may be a sort of 'window' into what work might, or should be, if it still represents, as we believe it does, a major way of discovering and pursuing meaning, identity and happiness. What some coworking experiences seem to showcase is the possibility to reconstruct the relationship between the individual identity and the collective identity, one where the two may actually strengthen each other rather than clash. Such possibility must not be necessarily confined to 'special' cases. We argue that some coworking experiences trigger useful inspirations about the possibility of re-imagining work in a broader sense and, at least potentially, in almost all work contexts, including traditional companies and organisations. In other words, in this paper we propose that observing coworking is a good way to reflect creatively about the complex relationship between people, work and organisations.

\section{What is coworking: a brief introduction and a case study}

Coworking is a recent phenomenon, but it is spreading and, even more importantly, it is evolving rapidly (Gandini, 2015). It originated as a relatively simple way for different workers, mostly freelancers or start-uppers, to share work spaces, services and equipment in order to decrease individual costs (Hood, 2015). That was the initial, simple motivation for the creation of early coworking initiatives. As we will see shortly, the phenomenon is turning into something much more interesting. Coworking 'centres', which are merely the spatial aggregation of freelancers interested in sharing resources and reducing costs, sometimes evolve into coworking 'organisations'. This is a loose term, not a specific concept. Our intent is to emphasise a sort of evolutionary continuum that can be observed, where at one end of the spectrum we find simple 'coworking centres' where coworkers share nothing more than spaces and equipment, and at the other end we find 'co-working organisations' where coworkers share an organisational (collective) identity and culture, and where organisational roles can be identified which are systematically engaged in defining a market strategy and even a structure of sorts.

Usually, there are at least three typical roles that seem to emerge: 
- the co-workers, that is, the individuals (freelancers) utilising spaces, equipment and services provided by the co-working organisation. More importantly, they participate in a number of collective activities and, de facto, constitute the working 'community'. Their participation can be varied in terms of continuity and intensity, and in relation to that they pay different tariffs or subscriptions

- the host(s), that is, those that initiate and often manage the co-working organisation (Spinuzzi, 2012), and can be assimilated to an entrepreneurial role; the hosts can also be co-workers themselves.

- the community manager(s), usually present only in larger co-working organisations, whom establish and manage both the relationships with the external market as an actual representative of the co-working organisation itself, and also play a relevant role within the organisation. It can be assimilated to a managerial responsibility of sorts. Sometimes, hosts also play the role of community managers.

The number of co-working organisations is increasing rapidly, all around the world (Moriset, 2014; Badiali, 2014). But the most interesting phenomenon is not just their numerical rise. Far from being just a way to share spaces, services and equipment, and to reduce costs, many well developed co-working organisations are also radically changing their purpose and raison d'etre (Bouncken and Reuschl, 2018). From the point of view of individual co-workers, these organisations increasingly represent a place where valuable relations and collaborations develop, as they allow participants to be involved in positive transformative work experiences (Pine and Gilmore, 1999), which enrich them in personal and professional terms. From the point of view of the co-working organisation as a whole, basic services and activities tend to evolve into increasingly complex ones, all the way to becoming, in the most advanced cases, an actual proactive collective subject, with a clear market strategy aimed at providing advanced, customised services to external customers, including companies and other organisations, based on the competences and skills possessed by the co-workers.

It is much easier to illustrate such evolution through a brief example. We will utilise the Warehouse Co-Working Factory case, a co-working organisation founded just a few years ago by converting an old textile factory (hence the name) in an area between Marotta and Mondolfo, in the centre-north of Italy, close to Pesaro-Urbino. At the time of our study, this organisation included 22 open space work spaces, 3 offices, a meeting room and several acoustically isolated small rooms for private skype and phone calls. There is also a relax/reading area, a coffee shop and a technical area (with printers, scanners and other equipment). There are about 50 freelancers that, overall, attend this co-working organisations, either intermittently or with more continuity. An interesting aspect is that, regardless of its small size and very recent history, this organisation is structured as an actual community, consistently with the original strategic vision of the two hosts/founders. This characteristic has very intriguing consequences, in terms of initiatives for both the co-workers themselves and also external subjects. Let us see a few examples of both types.

In terms of internal initiatives, besides the most common packages of basic services for coworkers (workstations, connectivity, meeting spaces etc) there are also experiential initiatives, with both social and professional connotations. As an example, there are seminars and workshops where attendees have the chance to learn specific professional skills. These events are mainly directed towards 'internal' co-workers, but they are open to external subjects which are interested in becoming members of the community, and are usually held by one or more 
co-workers themselves, who assume a 'teaching' role. The goal is typically to facilitate crossfertilisation and mutual learning between participants, but also to generate and support potential collaborations between co-workers with different competences and backgrounds and, most importantly, to generate new ideas and projects to be proposed to the open market in a sort of 'bottom-up', 'emergent' way. This example shows that the co-working organisation facilitates not only the individual growth and learning through both the daily informal interactions and structured, formal events, but it also functions as an actual creative hub capable of generating entrepreneurial ideas and opportunities for the co-workers themselves. Indeed, research shows that co-working organisations, by developing collaborative networks, can promote local entrepreneurship (Fuzi, 2015).

At the same time, Warehouse systematically promotes initiatives specifically aimed to external subjects, potential customers or partners, in order to develop a dense network of relations with significant actors such as companies, institutions, associations etc. The goal is to identify and develop partnerships for specific activities and projects aimed at extending the set of opportunities for the co-workers. From the potential customers' point of view(for example, other companies) this approach is consistent with the rapidly increasing use of 'staff on demand' (Pompa, 2017) in some of the most innovative organisations, often called 'exponential organisations' according to the model proposed by Salim Ismail (2014). Thus, coworking organisations seem to provide external companies a more structured, reliable and goal-driven way to extend their utilisation of staff on demand (Ross and Ressia, 2015).

At Warehouse, one initiative specifically aimed towards external potential partners and customers is called 'BarCamp' (participants informally call these events 'non conferences'). They are usually completely open and free, with the goal to attract entrepreneurs and professionals, mostly from the surrounding region, and inform them about the competences, ideas and solutions that present within the community of co-workers may provide. The results are quite promising. As an example, a local entrepreneurial association (CNA) was involved in order to organise, within Warehouse, an instance of the event Digitaly Imprese, promoted by Google and Amazon, which brought several small and medium local companies to interact with the co-working community, leading to several consulting projects where many coworkers were involved. BarCamp events are free and open exactly because they are aimed at opening new scenarios on specific problems and challenges for the companies. They can also focus on more specific technical themes, with a limited number of participants, and in that case a fee may be requested to external participants. What these examples show is that Warehouse is evolving towards the creation of a significant portfolio of technical/consulting services and ideas which may either emerge freely and spontaneously from the community of co-workers, but also may be specifically designed by the co-working organisation as a whole according to a clear overall strategy.

Another interesting example is the project Geronimo, again stemming from a collaborative effort with an external agency, in which several co-workers acted as teachers with a specific focus on skills and competences that are crucial for potential entrepreneurs interested in creating new companies. Other similar initiatives are emerging rapidly.

In general, it is worth noting that Warehouse, similarly to many other advanced co-working experiences, is trying to build a solid collaborative network, both inside the co-working organisation itself (the 'community') and with other organisations, including local authorities and institutions, Universities and even other co-working communities. Significant examples 
are collaborations between Warehouse and Agora, in Berlin (Germany), a very advanced coworking organisation, and Poligon, also a very advanced co-working case in Slovenia.

Besides representing a remarkable phenomenon in itself, in this paper we would like to propose a reflection on what these advanced co-working experiences such as Warehouse may suggest, in general terms, for work organisation and human resource management in more traditional settings such as business companies. We believe that the reason for the success of advanced co-working experiences mainly concern the fact that they insist on some organisational possibilities that are not yet explored and practiced enough in traditional companies, even though there are relevant examples of companies that are taking significant steps towards organisational arrangements that seem to be better suited for knowledge workers (Kubatova, 2014). These possibilities found a very fertile context in co-working settings, but we believe that, with some adjustments, similar working conditions could be recreated even in other settings. Indeed, the aspects we would like to focus on in this paper are among the most universal and pervasive one could imagine: people motivation, work organisation and leadership.

\section{Co-working as an inspiration to rethink work organisation: employees' motivation}

The most advanced co-working organisations seem to emphasise participants' work motivation as a key ingredient of their success. On the one hand, literature clearly shows that work motivation cannot be reduced to neither relatively simple stimulus-response mechanisms, nor to the use of mere incentives and sanctions in order to increase effort, commitment and performance. It is quite clear that human motivation, and specifically work motivation, is much more complex than that. While decades of research and studies have provided ample evidence of such complexity, one must admit that the proliferation of new concepts and terms, especially within the organisational and managerial literature, while sometimes proves to be useful because different concepts may help illuminate different sides or subtleties of the same general phenomenon, it may also generate confusion and unnecessary literature heterogeneity (just to mention a few of such variety of ideas which are undoubtedly 'related' to motivation: engagement, empowerment, organisational citizenship, organisational commitment, the list could go on). Here we explicitly choose to focus on what is probably the most classic distinction that seems to be not only extensively studied and documented, but also fundamental for the understanding of motivational processes: the intrinsic and the extrinsic aspects of human motivation (Ryan and Deci, 2000; Sansone and Harackiewicz, 2000), with a specific focus on work motivation.

The first general observation is that while it is abundantly clear that extrinsic motivation plays just a partial role, although important, in determining the relationship between man and work in motivational terms, and even though it implies a number of potential risks, disadvantages, contradictions and uncertainties, still today the vast majority of motivational mechanisms used by companies is based mostly, and sometimes solely, on extrinsic factors. We don't want to dwell here on the reasons for that, and we fully acknowledge that excellent counter-examples also exist. But it seems useful to recognise the widespread underestimation of the relevance of intrinsic motivation and, on the flip side, the general overestimation of the extrinsic mechanisms' effectiveness. The experience of advanced co-working organisations, on 
the contrary, seems to sharply emphasise the intrinsic aspects of the human work experience. We can specifically focus on four different aspects.

First, the strong sense of autonomy and self-determination that characterise co-workers is certainly a central aspect of their work experience. Autonomy is, obviously, a constitutive element of co-working as a general idea, even in less advanced cases. Members of co-working experiences are, by definition, freelancers, that is, autonomous workers. It is less obvious to observe the fruitful co-existence of such autonomy, even though managed and exercised in variable ways, and the presence of significant interdependences not only between individual co-workers, but also between co-workers and the co-working community as a whole. The emerging message is quite simple: control, hierarchy and supervisory are not the only possible, effective answers to interdependence. On the contrary, it is seems possible to reconcile dense, complex interdependencies with significant levels of individual autonomy, a reconciliation which is rarely observed in most traditional organisations. The consequence of such coexistence is not necessarily chaos or opportunism, as it is more or less implicitly suggested within the traditional dichotomic visions between autonomy and control. A different consequence of higher autonomy, if the right conditions are set, is precisely an increase in intrinsic motivation, as classic literature suggests (Deci et al., 1989; Deci and Ryan, 2000). And intrinsic motivation is a key ingredient for containing or even eliminating excessive opportunism, individualism and chaos, as we will argue shortly.

Second, co-working experiences are rich in terms of opportunities for personal and professional growth, learning, acquisition of new relations, and development of new individual and collective knowledge. On the one hand, autonomy helps explaining why this happens. When people are (more) free to act, often they will try to perform activities and tasks not only to fully utilise their existing competences and skills, but also to acquire and develop new ones (Hobfoll, 1989). So, autonomy, besides generating intrinsic motivation, also helps in generating growth opportunities. On the other hand, the social structure and the proximity of co-workers also facilitate the creation of unintended learning occasions (Parrino, 2015), which appear to be 'casual' but, in reality, are actually not, because they happen within an organisational context which is specifically designed to become Schelling points and facilitate mutual learning (Waters-Lyncs and Potts, 2017). Personal growth and learning are also a key component of intrinsic motivation.

Third, we must consider that co-working organisations, thanks to the high level of autonomy and the various, diverse opportunities for growth and learning, allow a better coupling between work activities and personal attitudes, passions and interests. In other words, the coworking 'structure' seems to facilitate what is usually quite difficult to achieve in traditional organisations, at least for most people, that is a careful consideration of the uniqueness of each individual in designing and attributing work activities. In co-working settings, jobs are largely 'designed' (indeed, chosen) by the subjects themselves so that they will be more compelled to work on activities and projects that are more interesting and meaningful for them. At the same time, feeling part of a 'community' may also add depth to the meaningfulness of their work experience (Spreitzer et al., 2015). Not only, but the variety of stimuli that co-working organisations provide may also help co-workers to discover new interests, new passions that they didn't know about. In 2012, Gandia found in a survey that involved about 1500 freelancers that the top two reasons for freelancing are 'more freedom and flexibility' - in other words, autonomy - (28\% of respondents) and the ability to 'follow my passion' ( $23 \%$ of 
respondents), while 'a source of additional income' was chosen only by $7 \%$ of respondents. Again, the consonance of one's job with his/her own true passions and interests is also a key component of intrinsic motivation (Amabile, 1998). This result emphasises the relevance of passion (and, again, autonomy) as a driver of freelancers' intrinsic motivation, while extrinsic motivation (incentives and income) seems to be far less relevant.

Finally, we should also consider the idea that intrinsically motivating jobs are the ones where individuals understand or, even better, agree with the goals, the purposes and the values that they pursue through their work activities. In this respect, co-working organisations not only allow individuals to autonomously contribute and participate to the means of production, but also to the definition of goals and missions. Again, as goal setting theory clearly shows (Elliot and Harackiewicz, 1994) participation in defining goals has a strong effect on intrinsic motivation.

Overall, the social structure of advanced co-working experiences, because of the way it tends to evolve, seems to produce almost 'naturally' a high level of intrinsic motivation, in all its key components. The picture we are painting may look exceedingly positive and optimistic. Admittedly, there may be significant problems and difficulties. When autonomy is high, the risk of insufficient coordination or, worse, the danger of widespread opportunism is very real. Whether risks and problems prevail on potential advantages is an empirical question, which is probably dependent on a variety of contextual factors. What works well in some organisational settings may turn out to be a disaster in others, but this is also true for any organisational arrangement, even the most traditional ones. So, we are not claiming that advanced co-working experiences provide universal, fool-proof principles. We argue, instead, that intrinsic motivation, if truly elevated and present within an organisational setting designed to continuously regenerate it, may act as a powerful 'vaccine' against opportunistic, centrifugal dangers of highly informal, horizontal, variable coordination practices. When people are highly intrinsically motivated, they become less inclined to opportunism simply because they know that overly individualistic behaviours may contribute to destroy the same work context from which they obtain significant satisfaction and results. Not only, but they will be more willing to act, or even sacrificing part of their short-term personal interests, in order to maintain the same characteristics of the work context that make it so motivating and compelling for them in the long term. For example, they might gladly accept to give up part of their autonomy in order to ensure better coordination within a certain project, while knowing that such 'step back' is only temporary, voluntary, instrumental to a goal that they desire to achieve, and not imposed by a formal hierarchy, but the outcome of a shared evaluation about the an objective 'need' to improve coordination. Such a 'vaccine' may not be always sufficient to avoid negative aftermaths. But co-working experiences seem to suggest that there is ample room for experimentation, even in more traditional work settings, in order to seriously rethink, or even redesign the intersection between work organisation, individual motivation and coordination mechanisms, towards a direction which is geared neither towards better control and compliance mechanism, nor toward persuading or communicating in a better way top-down decisions, but toward investing in peoples' sense of responsibility, awareness, self-fulfillment and, in the end, the construction of a better self-identity. 


\section{Co-working, job design and leadership}

Traditional job design approaches, even the more progressive practices of job enrichment and job enlargement, seem to bear a certain 'original sin', an underestimation of people's ability to contribute positively to the conception and design of their own work. Tasks are always designed and attributed by 'others', typically by the managerial hierarchy or staff units that are perceived as outsiders (in relation to the actual, daily work practices). Hence, they are perceived to be imposed - because they are! - to employees, no matter how hard organisations try to communicate, listen, 'enlarge' or 'enrich' jobs. Even a very enlarged, enriched job is still an imposition, if employees do not actually and directly participate to its design. The rationale for such an approach is simple: only by observing 'from above' it is possible to have a birdeye, integrated vision of work processes, interdependencies and requirements and that are necessary to achieve the best collective outcome. Those who do not have such a vision - the people at the lower levels of the hierarchy - may, at best, suggest marginal adjustments. One might argue that such an approach is part of the Tayloristic heritage, or one could also argue that it's just common sense, a mere fact of life, which cannot be avoided.

However, if such logic could not be avoided in any way, it would be difficult to explain how advanced co-working organisations may possibly function properly and even thrive. Job design, as defined in its classic conceptualisation, does not exist in such organisations. Also, this is true not only for co-working organisations. Other companies and organisations are experimenting, with very good results, other forms of work organisation, also based on a significant, sometimes radical rethinking of job design (Kubatova, 2014). Literature shows that a more 'bottom-up' approach to work organisation may actually increase individual and organisational performance, well-being, satisfaction (Pink, 2009). Obviously, a 'cultural' shift, a new awareness of what managers and leaders should do in order to change the organisational logic in such a dramatic way is absolutely crucial (Hill et al., 2014).

An example can be found in studies devoted to proactivity at work, that is, behaviours through which employees (at all levels, not necessarily just blue collars or low level white collars) become protagonist and take initiative in order to change (re-design) their own jobs, even in relation to non-trivial aspects (Grant and Ashford, 2008). Sometimes such behaviours are tolerated or even accepted by higher level managers. Other times this is not the case, as employees may even 'break the rules' in order to 'conquer' autonomy and take responsibility for the definition of their jobs. Several concepts are available in current literature that describe different aspects of proactive behaviours in the workplace. One of the most studied, even in empirical research, is the construct of 'job crafting', which is a set of behaviours through which employees choose to autonomously modify tasks, relations and meanings of their job. For example, they may seek new challenges, seek new resources, avoid hindering tasks etc. Literature on job crafting, which largely stemmed from the seminal paper by Amy Werznewski and Jane Dutton (2001), is gradually but clearly showing that allowing or even facilitating job crafting does not necessarily imply negative consequences in terms of individual or organisational performance. On the contrary, most evidence show a number of positive outcomes (Rudolph et al, 2017). At the individual level, more satisfaction, commitment, motivation, identification and performance can be observed. At the organisational level, better problem solving, creativity, knowledge creation and dissemination, and general performance can also be observed. More research is needed, but most signs seem to point toward the same direction. There's also some evidence of possible 
negative consequences of job crafting, but it seems that these are mostly due to the inability of organisations and/or leaders to create the right conditions for job crafting to fully develop its potential. Having the right conditions is absolutely crucial. The role of leadership is key, as leaders should focus on facilitating exchanges, dialogue, participation to decision making - in other words, leadership style should be similar to what literature calls 'promotive leadership', rather than 'restrictive leadership' (Berdicchia and Masino, 2018). It is essential that leaders aim at creating an organisational, cultural and social context which promotes proactivity rather than imposing specific behaviours and ideas through procedures and standards. Indeed, methodological leadership (rather than substantial leadership, which is what more commonly, and mistakenly, leadership is associated to) is probably the most important shared characteristic of many among the most innovative organisations, in all sectors (Hill et al., 2014).

All this leads us back to co-working, because what happens in the most advanced co-working experiences is precisely what literature on job crafting and on innovative organisations seem to propose. Co-working is, indeed, a work situation in which co-workers are not only allowed, but even encouraged and facilitated to co-design their job - in other words, to be very advanced 'job crafters'. In co-working organisations, leaders (hosts, community managers and other informal leaders) create the right work conditions rather than impose procedures, tasks or even specific goals. It is a blend of high autonomy and responsibility, continuous exchange and dialogue opportunities between highly heterogeneous competences, information sharing, monitoring and developing opportunities from the outside, leadership roles that are not crystallised but change dynamically depending on the specific activities and projects under development - which is what Laszlo Bock, former head of HR in Google, calls 'emergent leadership' (Bock, 2015).

It is worth noting that we are neither describing an absence of leadership, nor a sort of anarchy. On the contrary, co-working organisations do require specific conditions and need precise 'rules' of the game, which have to be understood, accepted and internalised by the protagonists, and leaders have the responsibility of defining and implementing them. The core idea is the separation between methodological leadership from substantial leadership. The former is somewhat 'fixed', it is connected to a specific expertise, which is methodological/organisational, and it has the crucial role of defining the work context and its culture, its norms, its 'rules'. The latter, on the contrary, needs to be variable and decentralised because it has to change depending on the nature of each project or activity, as it is related to the specific competences that are contingently required. In a sense, the methodological leader is not the main character, as s/he is the one that 'sets the stage' so that the real protagonists are put in the condition to perform at their best. Hence, advanced co-working experiences seem to suggest the possibility of escaping from the never-ending pendulum between traditional solutions, where job design is a prerogative of the hierarchy, and utopian solutions, where job design is a sort of purely bottom-up, emergent phenomenon. Instead, the very idea of 'job design' - which is, the idea of predetermining jobs - seems to be abandoned altogether, in the sense that the multidimensionality of the problem of work organisation is finally recognised, and its solution is not found in some sort of magical balance between centralisation and decentralisation, but in a variable combination of different 'kinds' of leadership roles, which is made possible by a culture that emphasises intrinsic motivation, autonomy and responsibility. 


\section{Final remarks}

While in previous paragraphs we tried to disentangle some of the many elements that make advanced co-working experiences interesting and inspiring, one should recognise that the key ingredients - intrinsic motivation, proactivity and leadership - are tightly connected with each other. If intrinsic motivation is lacking, then the possibility of organising work by leveraging proactivity and job crafting may easily generate opportunistic or counter-productive behaviours. Intrinsic motivation is what persuades people not to behave in a way that may damage the work context in which they are thriving. On the other hand, if autonomy is limited or even not permitted, then proactivity and job crafting cannot fully develop, because proactive behaviours could be seen as in conflict with the coordination needs, with the incumbent leadership or with other members, hence workers would be less willing to take chances for the fear of being sanctioned. Finally, if an effective, methodological leadership is lacking, then the very conditions that facilitate the development or intrinsic motivation and proactive behaviours would be missing, because it is precisely those social, cultural and organisational conditions created and facilitated by a methodological leadership that make this new way of thinking work organisation even possible. In other words, all three elements seem to be necessary, and that is why advanced co-working experiences represent interesting organisational 'experiments', as the people involved (hosts/leaders and co-workers) seem to embrace exactly these three principles: co-workers appear to be highly intrinsically motivated, there is a widespread culture for autonomy and proactivity, and hosts act as methodological leaders while not interfering with substantial matters.

Second, we need ask to what extent this kind of organisational logic, however 'inspirational', can be actually 'exported' to more traditional organisational settings, outside of the coworking 'world'. More specifically, one should ask what are the kinds of work contexts in which this logic may have good chances to succeed. This is an empirical question, and research is needed in order to find credible answers. However, we can provide some clues that may be helpful to reflect on this issue. On the one hand, it seems plausible that co-working-like work arrangements are more suitable for companies and organisations that need significant contributions from their employees in terms of creativity, problem solving and flexibility. On the contrary, where activities are more easily and effectively predetermined and standardised, the advantages may seem to vanish or to be irrelevant. While this may be a valid, general point, a word of caution seems necessary. There are cases (Zappos is a famous one) showing that allowing employees to enjoy more autonomy and creating an intrinsically motivating work environment may bring about significant, unexpected benefits even when work activities appear to be, ex ante, pretty standardised. The value that motivated, autonomous, proactive employees may bring even in otherwise apparently stagnant work environments should not be underestimated. Also, the need for continuous improvement or microinnovation is pervasive, even in industries and organisations where creativity does not seem to be the main driver. In the end, it seems that only genuine experimentation may provide a definitive answer about what advantages co-working-like work arrangements could bring about in any specific context.

Rather, the problem may often lie in a managerial culture that often seems to hinder or even reject innovative solutions to work organisation problems - a cultural heritage that may influence not only managers and consultants, but even workers themselves. It's a mindset that sometimes may just simply translate into insufficient willingness to experiment, to try new 
things, to test new ideas in order to identify, in each specific context, unique organisational solutions to ultimately improve the current state of affairs. Laszlo Bock, in Google, embraced organisational experimentation as one of his most important mantras. This, of course, does not mean that Google's solution are easily exported to other organisations, but what should be exported is the core belief that organisational innovation is a key element for the company's competitive advantage, that a continuous effort should be devoted to it, and contextual experimentation is the best, maybe the only way to discover new possibilities. By doing so, Bock perfectly demonstrates the importance of his methodological leadership, clearly separated from substantive leadership. On the contrary, in many other traditional settings, it seems implicit that innovation in work organisation is only marginally important, if at all, because everything that's relevant is already understood and implemented, and when organisational innovation is carried out is only driven by technological change. We believe that this is a misleading attitude. There is a lot of room for improving work organisation, not only because technology is providing new opportunities, but also because available knowledge about human psychology and behaviour, especially in work settings, strongly suggests that we can imagine very different ways to organise work in order to combine organisational performance advantages and individual satisfaction and well-being. Coworking organisations may represent a sort of peculiar, small 'window' into an interesting, inspiring array of mostly untapped opportunities even for traditional companies. As long as work will be a major path to individual identity and happiness, such little 'window' may be more important than what it may appear to be.

\section{Reference list}

Amabile, T.M. (1998), "How to kill creativity", Harvard Business Review, 76 (5): 76-87.

Badiali, E. (2014), Work Together - Right Now. Coworking, cooperazione e creatività, Rapporto di ricerca, Fondazione Ivano Barberini, Bologna.

Berdicchia, D., and Masino, G. (2018), "Leading by leaving: Exploring the relationship between supervisory control, job crafting, self-competence and performance", Journal of Management and Organization, 1-19.

Bock, L. (2015), Work rules!, New York: Hachette Group.

Bouncken, R. B., and Reuschl, A.J. (2016), “Coworking-spaces: how a phenomenon of the sharing economy builds a novel trend for the workplace and for entrepreneurship", Review of Managerial Science, 12 (1): 317-334.

Deci, E.L., Connell, J.P., and Ryan, R.M. (1989), "Self-determination in a work organization”, Journal of Applied Psychology, 74 (4): 580-590.

Deci, E.L., and Ryan, R.M. (2000), "The "what" and "why" of goal pursuits: Human needs and the self-determination of behavior", Psychological Inquiry, 11 (4): 227-268.

Elliot, A.J., and Harackiewicz, J.M. (1994), “Goal Setting, Achievement Orientation, and Intrinsic Motivation: A Mediational Analysis", Journal of Personality and Social Psychology, 66 (5): 968-980.

Fuzi, A. (2015), “Co-working space for promoting entrepreneurship in sparse regions: the case of South Wales", Regional Studies, Regional Science, 2 (1): 462-469. 
Gandia E. (2012), “Freelance Industry Report”, [online], Scribd,

Gandini, A. (2015), “The rise of coworking spaces: A literature review”, Ephemera: theory $\mathcal{E}$ politics in organization, 15 (1): 193-205.

Grant, A.M., and Ashford, S.J. (2008), "The dynamics of proactivity at work", Research in Organizational Behavior, 28: 3-34.

Hill L., Brandeau G., Truelove E., and Lineback K. (2014), Collective genius: the art and practice of leading innovation, Boston: Harvard Business Review Press.

Hobfoll, S.E. (1989), "Conservation of resources. A new attempt at conceptualizing stress", American Psychologist, 44 (3): 513-524.

Hood, C. (2015), "Building communities: Lessons in co-working for the enterprise world", Corporate Real Estate Journal, 4 (3).

Ismail, S. (2014), Exponential Organizations, New York: Diversion Books.

Kubatova, J. (2014), “The Cause and Impact of the Development of Coworking in the Current Knowledge Economy", in Vivas, C. Sequeiro, P. The Proceedings of the 15th European Conference on Knowledge Management. London: Academic Conferences and Publishing International Limited, 571-577.

Moriset, B. (2014), "Building new places of the creative economy. The rise of coworking spaces", in Proceedings of the 2nd Geography of Innovation, International Conference 2014, Utrecht, The Netherlands: Utrecht University.

Oldham, G.R., and Hackman, R. (2010), “Not what it was and not what it will be: the future of job design research", Journal of Organizational Behavior, 31 (2-3): 463-479.

Parrino, L. (2015), "Coworking: assessing the role of proximity in knowledge exchange", Knowledge Management Research and Practice, 13 (3): 261-271.

Pine, B.J., and Gilmore, J.H. (1999), The Experience Economy. Work is Theatre E Every Business a Stage, Boston: Harvard Business School Press.

Pink, D. (2009), Drive: the surpising truth about what motivates us, Penguin, New York.

Pompa, L. (2017), “From the Unusual to the Useful: Digital Platforms and Co-Working Spaces as Instruments for Human Resource Management in Emerging Economic and Organizational Environments", International Journal of Business and Management, 12 (8).

Ross, P., and Ressia, S. (2015), "Neither office nor home: coworking as an emerging workplace choice", Employement Relations Record, 15 (1): 42-57.

Rudolph, C.W. Katz, I.M., Lavigne, K.N., and Zacher H. (2017), "Job crafting: A meta-analysis of relationships with individual differences, job characteristics, and work outcomes", Journal of Vocational Behavior, 102: 112-138.

Ryan, R.M., and Deci, E.L. (2000), "Intrinsic and extrinsic motivations: Classic definitions and new directions", Contemporary Educational Psychology, 25: 54-67.

Sansone, C., and Harackiewicz, J.M. (2000), Intrinsic and Extrinsic Motivation: The Search for Optimal Motivation and Performance, London: Academic Press.

Spinuzzi, C. (2012), "Working alone together. Coworking as emergent collaborative activity", Journal of Business and Technical Communication, 26 (4): 399-441. 
Spreitzer, G., Bacevice, P., and Garrett, L. (2015), "Why people thrive in coworking spaces", Harvard Business Review, September issue, 28-30.

Waters-Lynch, J., and Potts, J. (2017), “The social economy of coworking spaces: a focal point model of coordination", Review of Social Economy, 75 (4): 417-433.

Wrzesniewski, A., and Dutton, J. E. (2001), “Crafting a job: Revisioning employees as active crafters of their work", Academy of Management Review, 26 (2): 179-201. 